\title{
DETECCCÃo de ALTeraÇões em exame físico da Pele DA REGIÃO DE APOIO DE PACIENTES SUBMETIDOS PRO- LONGADAMENTE A UM MESMO DECÚBITO
}

\author{
Maria Goretti Angarten* \\ Maria Lúcia Forte dos Santos**
}

\begin{tabular}{l|l|}
\cline { 2 - 2 } & RePEn/06 \\
\hline
\end{tabular}

ANGARTEN, M.G. e Colaboradora - Detecção de alterações em exame físico da pele da regiāo de apoio de pacientes submetidos prolongadamente a um mesmo decúbito. Rev. Bras. Enf.; DF, 33 : 443-452, 1980.

\section{INTRODUÇÃO}

A prevenção e o tratamento de escaras de decúbito têm sido um grande desafio para o pessoal de enfermagem. As pesquisas feitas para a melhor compreensão da gênese das escaras de decúbito são em grande número e estão bem reunidas em trabalho de revisão de BERECEK ${ }^{1}$, assim como de COMARÚ e CAMARGO 2. De acordo com esses trabalhos, o principal fato que explica o início de uma escara de decúbito é a isquemia da região de apoio, uma vez que esses estudos experimentais mostram que a pressão nessas regiões é superior à pressão da perfusão dos tecidos. Esses trabalhos também concluem que, além da intensidade da pressão, a duração dela representa um fator associado à produção de escaras de decúbito, bem como a distribuição do peso origina intensidades de pressão variáveis ao longo da superfície de apoio, sendo obviamente mais intensa nas regiōes de proeminências ósseas.

As pacientes que são tratadas com radioterapia, usando a técnica de radium moldagem, são submetidas a muitas das condições experimentais descritas nesses trabalhos.

Este nosso trabalho tem como objetivo verificar a incidência de escaras de decúbito nessas pacientes através de estudo controlado que permita dar contribuições à pesquisa da gênese das escaras de decúbito.

A revisão da literatura que ora resumimos consta de trabalhos que estudaram incidência de escaras de decúbito em pacientes hospitalizados.

* Enfermeira Supervisora do Hospital Osvaldo Cruz e Coordenadora do Grupo de Atualização Científica do Serviço de Enfermagem do H:spital Osvaldo Cruz, São Paulo. ** Enfermeira da 5." Unidade de Internação do Hospital Osvaldo Cruz, São Paulo. 
ANGARTEN, M.G. e Colaboradora - Detecção de alterações em exame físico da pele da região de apoio de pacientes submetidos prolongadamente a um mesmo decúbito. Rev. Bras. Enf.; DF, 33 : 443-452, 1980.

WILLIANS 7 estudou 26 pacientes não ambulantes sob 21 aspectos e sete deles $(26,92 \%)$ desenvolveram escara. A própria autora comenta que diversos fatores interferiram no aparecimento de escara (por exemplo, cuidados de enfermagem não padronizados) e que houve fatores coincidentais na pequena população estudada, não permitindo conclusões. A maior correlação entre um fator estudado e o desenvolvimento de escara foi encontrada quando se considerou o peso corpóreo, cujo índice de correlação foi de $52,2 \%$ (a maior incidência de escara esteve associada a peso menor).

RUBIN et col ' , em trabalho retrospectivo, estudaram em hospital geral, por um período de nove meses, os 18.000 pacientes admitidos, dos quais 67 deles já tinham escara na admissão, 83 desenvolveram escara durante a internação e 112 tinham escara, porém não havia anotação se ela havia se desenvolvido antes ou após a internação. Dos pacientes que tiveram escara, 96 tinham diagnóstico não registrado, 81 de debilidade outra que não por doença maligna, 22 tinham incontinência, 21 problemas ortopédicos, 18 tinham cancer, 16 pacientes tinham patologias cirúrgicas e 8 diabetes.

GERSON 3 estudou 5.648 pacientes de três hospitais de tratamento de afecções agudas, cuja média de permanência do paciente era de 13 dias e cuja média de idade dos pacientes era de 49,5 anos. Os pacientes foram submetidos a exames diários e $2,69 \%$ deles desenvolveram escara, $67,9 \%$ anos foi a média de idade dos pacientes que desenvolveram escara. Três eram os principais diagnósticos desses pacientes: neopiasias, doenças do aparelho circulatório e trauma por acidentes.

LOWTHIAN 5 realizou estudo da prevalência de escaras de decúbito em
186 pacientes de um hospital ortopédico e encontrou prevalência de $7 \%$ de escara (13 pacientes). Dos 29 pacientes com 70 anos ou mais, quatro $(13,8 \%)$ tiveram escara de decúbito. A baixa prevalência de escara encontrada nessa pesquisa está provavelmente relacionada à média de idade da população estudada $(44,2$ anos) e à ênfase do pessoal de enfermagem especialmente na prevenção de escara de decúbito.

Desses estudos fica a idéia de que a existência de escara de decúbito é variável, obedecendo características de cada população; assim considerando, em hospital geral há incidência variável de $2,69 \%$ a $10 \%$, em hospital ortopédico $7 \%$ e em um grupo de pacientes não ambulantes a alta incidência de $26,9 \%$. No entanto, dentro de cada um desses estudos, há subgrupos de pacientes em que a incidência de escaras é maior ou menor do que a do grupo como um todo. Talvez as características que identificam alguns desses subgrupos estejam implicadas na gênese das escaras de decúbito; porém em nenhum deles existe, dadas as características dos grupos, possibilidade de conclusão.

\section{DESENVOLVIMENTO}

Casuística e método

A população do presente trabalho constitui-se de 26 mulheres internadas, consecutivamente, no Hospital Osvaldo Cruz, a partir de 18 de janeiro de 1980 , com diagnóstico de carcinoma de colo de útero $(76,9 \%)$ e de carcinoma de endométrio $(23,1 \%)$, para tratamento braquicurieterápico utilizando a técnica de radium moldagem, por período de 40:00 a 80:10 horas.

Após a admissão, as pacientes foram submetidas a lavagem intestinal, banho, cateterismo vesical e orientação quanto ao tratamento. Depois da colo- 
ANGARTEN, M.G. e Cslaboradora - Detecção de alterações em exame físico da pele da região de apoio de pacientes submetidos prolongadamente a um mesmo decúbito. Rev. Bras. Enf.; DF, 33 : 443-452, 1980.

cação do radium, as pacientes ficaram em decúbito dorsal horizontal, com travesseiro baixo e sem outros coxins. O tipo de colchão utilizado foi o de espuma, recoberto de napa sobre cama de estrado de tábua. Houve possibilidade de movimento dos membros superiores, e ativo, porém limitado, dos membros inferiores; no entanto, não houve possibilidade de movimentos do tronco. Durante o tratamento, as pacientes receberam dieta sem resíduos e não houve manipulação que as fizesse sair do decúbito descrito.
Todas as pacientes foram submetidas, na admissão, a exame da pele da região dorsal pelas autoras que as classificaram em um de quatro grupos. Três dos grupos foram constituídos de pacientes com escaras de decúbito de $1 .^{\mathrm{a}}, 2 .^{\mathrm{a}}$ ou $3 .^{\mathrm{a}}$ fase, de acordo com a classificação usada por RUBIN e col.6 (tabela 1). E no quarto grupo estavam as pacientes cuja pele não apresentavam escaras, porém podiam estar presentes verrugas, cicatrizes, lesões actínicas e maceração interglútea, o que ocorreu em $19,2 \%$ das pacientes.

\section{TABELA 1}

Classificação das escaras de decúbito utilizadas por RUBIN e col. "

1. ${ }^{\text {a }}$ fase - escara incipiente, área avermelhada com pele íntegra.

2. ${ }^{a}$ fase - escara com formação de bolha ou úlcera.

3. ${ }^{\text {a }}$ fase - escara com necrose, odor fétido, ou ambos.

De dez minutos a três horas, após a retirada do aparelho de radium moldagem, a pele da região dorsal das pacientes era examinada pelas autoras, segundo o procedimento do exame inicial.

Todas as pac:entes que apresentavam mais de uma escara e de fases diferentes foram classificadas como pertencendo ao grupo de escara de fase mais avançada.

Foram coletados os dados de idade, cor da pele, peso, altura e duração do tratamento das pacientes observadas. Os dados de peso, altura e idade foram utilizados para se calcular, através da tabela do INSTITUTO DE RESSEGUROS DO BRASIL 4, a relação peso/peso ideal de cada paciente.

\section{RESULTADO}

A idade das pacientes variou entre 41 e 84 anos, com distribuição por faixa etária mostrada na tabela 2.

21 pacientes eram de cor branca; 2 de cor preta e 3 de cor parda.

A distribuição das mulheres, segundo a relação peso/peso ideal, está resumida na tabela 3 . 
ANGARTEN, M.G. e Colaboradora - Detecção de alterações em exame físico da pele da região de apoio de pacientes submetidos prolongadamente a um mesmo decúbito. Rev. Bras. Enf.; DF, 33 : 443-452, 1980.

TABELA 2

Distribuição das pacientes por faixa etária

\begin{tabular}{|ccc|c|}
\hline idade (em anos) & $n^{8}$ de pacientes \\
\hline 40 & $\vdash$ & 50 & 5 \\
\hline 50 & $\vdash$ & 6.0 & 10 \\
\hline 60 & $\vdash$ & 70 & 9 \\
\hline 70 & & & 2 \\
\hline Total & & 26 \\
\hline
\end{tabular}

TABELA 3

Distribuição das pacientes segundo a relação peso/peso ideal

\begin{tabular}{|c|c|c|c|}
\hline \multicolumn{3}{|c|}{ peso/peso ideal $(\%)$} & $n^{8}$ de pacientes \\
\hline 50 & r & 70 & 1 \\
\hline 70 & เ & 90 & 3 \\
\hline 90 & r & 110 & 9 \\
\hline 110 & r & 130 & 9 \\
\hline 130 & r & & 4 \\
\hline Tot & & & 26. \\
\hline
\end{tabular}

Quando consideradas as condições da pele prévias ao tratamento, 2.1 pacientes foram classificadas como tendo pele íntegra; 4 pacientes com escara de $1 .^{a}$ fase, e 1 paciente com escara de $2 .^{a}$ fase.

A distribuição das pacientes, em relação à duração do tratamento, está na tabela 4. 
ANGARTEN, M.G. e Cslaboradora - Detecção de alterações em exame físico da pele da região de apoio de pacientes submetidos prolongadamente a um mesmo decúbito. Rev. Bras. Enf.; DF, 33 : 443-452, 1980.

TABELA 4

Distribuição das pacientes, segundo o tempo de duração do tratamento

\begin{tabular}{|c|c|c|}
\hline dur & $\begin{array}{l}\text { tratamento } \\
\text { s] }\end{array}$ & $n^{8}$ de pacientes \\
\hline & 50 & 1 \\
\hline 50 & 60 & 0 \\
\hline 60 & .70 & 9 \\
\hline 70 & 80 & 15 \\
\hline 80 & & 1 \\
\hline Total & & 26 \\
\hline
\end{tabular}

Após o tratamento, dez pacientes foram classificadas no grupo de pele íntegra; sete no grupo de escara de $1 .^{\text {a }}$ fase; oito no grupo de escara de $2 .^{?}$ fase e uma no grupo de escara de $3 .^{a}$ fase.

Das quatro pacientes que tinham escara de $1 .^{\mathrm{a}}$ fase antes do tratamento, duas evoluíram para escara de $2 .^{a}$ fase e duas permaneceram com a escara de $1 .^{a}$ fase, porém em área maior. A única paciente que, anteriormente ao tratamento, tinha escara de $2 .^{a}$ fase se manteve com o mesmo tipo de lesão.

A distribuição das pacientes, em relação à durarão do tratamento e o resultado do exame após o tratamento, é mostrado na tabela 5. Deve-se notar que foram excluídas desta tabela as pacientes que tinham escara de decúbito no exame prévio ao tratamento. 
ANGARTEN, M.G. e Colaboradora - Deteç̧ão de alteraçōes em exame físico da pele da região de apoio de pacientes submetidos prolongadamente a um mesmo decúbito. Rev. Bras. Enf.; DF, 33 : 443-452, 1980.

TABELA 5

Distribuição das pacientes em relação à duração do tratamento e classificação da pele no exame após o tratamento

\begin{tabular}{|c|c|c|c|c|c|c|}
\hline \multirow{2}{*}{\multicolumn{2}{|c|}{$\begin{array}{l}\text { duração do } \\
\text { tratamento } \\
\text { (horas) }\end{array}$}} & \multicolumn{4}{|c|}{ classifićação } & \multirow[t]{2}{*}{ TOTAL } \\
\hline & & íntegra & $1^{a}$ fase & $2^{a}$ fase & $3^{a}$ fase & \\
\hline & -50 & 0 & 0 & 1 & 0 & 1 \\
\hline 50 & -60 & 0 & 0 & 0 & 0 & 0 \\
\hline 60 & -70 & 4 & 3 & 1 & 0 & 8 \\
\hline 70 & -80 & 6 & 2 & 2 & 1 & 11 \\
\hline 80 & & 0 & 0 & 1 & 0 & 1 \\
\hline Tota & & 10 & 5 & 5 & 1 & 21 \\
\hline
\end{tabular}

Ao considerarmos as 21 pacientes que não tiveram escara antes do tratamento e quando analisada a sua distribuição por faixa etária e em relação à ausência de escara ou a fase de escara após o tratamento, obtivemos os resultados da tabela 6 .

TABELA 6

Distribuição das pacientes por laixa etária e classificação da pele quanto ao exame da pele após o tratamento

\begin{tabular}{|c|c|c|c|c|c|c|c|}
\hline \multirow{2}{*}{\multicolumn{2}{|c|}{$\begin{array}{c}\text { faixa etária } \\
\text { (anos) }\end{array}$}} & \multicolumn{5}{|c|}{ classificação } & \multirow[t]{2}{*}{ TOTAL } \\
\hline & & integra & 1 fase. & $2 \stackrel{a}{-}$ & fase & $3^{\text {a }}$ fase & \\
\hline 40 & $\doteqdot 50$ & 3 & 1 & & 0 & 1 & 5 \\
\hline 50 & $r \quad 60$ & 5 & 2. & & 0 & 0 & 7 \\
\hline & $5 \quad 70$ & 2 & 2. & & 3 & 0 & 7 \\
\hline & 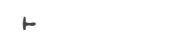 & 0 & 0 & & 2 & 0 & 2 \\
\hline \multicolumn{2}{|c|}{ TOTAL } & 10 & 5 & & 5 & 1 & 21 \\
\hline
\end{tabular}


ANGARTEN, M.G. e Colaboradora - Detecção de alterações em exame físico da pele da regiäo de apoio de pacientes submetidos prolongadamente a um mesmo decúbito. Rev. Bras. Enf.; DF, 33 : 443-452, 1980.

\section{TABELA 7}

Distribuição das pacientes por peso/peso ideal e classificação quanto ao exame da pele após o tratamento

Em relação à distribuição das pacientes pela relạão peso/peso ideal e a ausência de escara ou a fase de escara após o tratamento apresentamos os resultados encontrados na tabela 7 .

\begin{tabular}{|c|c|c|c|c|c|c|c|}
\hline \multirow{2}{*}{\multicolumn{2}{|c|}{$\begin{array}{c}\text { peso/peso ideal } \\
(\%)\end{array}$}} & \multicolumn{5}{|c|}{ classificaçäo } & \multirow[t]{2}{*}{ TOTAL } \\
\hline & & integra & $1-$ a & Fase & 2 a fase & 3 a fase & \\
\hline 50 & $\vdash \quad 70$ & 0 & & 0 & 0 & 1 & 1 \\
\hline 70 & - 90 & 2 & & 0 & 0 & 0 & 2 \\
\hline 90 & $+\quad 110$ & 4 & & 2 & 0 & 0 & E \\
\hline 110 & $\vdash \quad 130$ & 4 & & 2 & 2 & 0 & 3 \\
\hline 130 & $\vdash$ & 0 & & 1 & 3 & 0 & 4 \\
\hline TOTAL & & 10 & & 5 & 5 & 1 & 21 \\
\hline
\end{tabular}

\section{DISCUSSÃO}

Apesar do número de pacientes estudados, acreditamos que todos os raciocínios numéricos feitos estejam próximos da realidade, uma vez que os números encontrados são muito contrastantes. A incidência de pacientes que tiveram escara foi de $52,4 \%$ ne.ste trabalho, várias vezes maior que o encontrado na literatura 3567 . Este fato é explicado pelo tipo de tratamento ao qual as pacientes foram submetidas que. não permite a aplicação dos cuidados de enfermagem clássıcos de prevenção de escara.

Utilizamos a classificação de RUBIN e col. 6 por sua simplicidade e fácil aplicação prática. COMARÚ e col. ${ }^{2}$ classificam as fases de escara diferen- temente, considerando a $1 .^{\text {a }}$ fase o eritema que desaparece logo após a mudança de decúbito, constituindo-se então numa fase não detectável pelo método aqui empregado, dado que o exame da pele após o tratamento foi realizado pelo menos dez minutos depois da retirada do aparelho de radium moldagem. LOWTHIAN 5 não considera 0 eritema persistente (escara de $1 .^{\text {a }}$ fase de acordo com RUBIN e col. ${ }^{6}$ ) como fase da escara de decúbito, justificado pelo difícil diagnóstico de alteração da cor da pele quando o desenvolvimento dessas alterações não é acompanhado ou o paciente é de pele negra, exceto as alterações da necrose e da hemorragia. Esta colocação é pertinente para trabalhos de auditoria, condição diversa deste trabalho onde foi feito acompa- 
ANGARTEN, M.G. e Colaboradora - Detecção de alterações em exame físico da pele da regiāo de apoio de pacientes submetidos prolongadamente a um mesmo decúbito. Rev. Bras. Enf.; DF, 33 : 443-452, 1980.

nhamento das alterações da pele, realizando-se avaliação prévia e posterior ao tratamento. Em nosso trabalho, cinco pacientes não eram brancas (três de cor parda e duas de cor preta); em uma delas foi diagnosticada escara de $1 .^{\mathrm{a}}$ fase antes do tratamento que permaneceu inalterada até a segunda avaliação; as outras pacientes foram classificadas antes e depois do tratamento como tendo pele íntegra. Se levarmos em conta o argumento de LOWTHIAN 5, as conclusões do trabalho sofreriam pequena alteração no sentido de aumentar a incidência de escara, pois estas seriam menos diagnosticadas nas mulheres de cor escura. A variável cor não foi analisada em nenhum outro ponto deste trabalho devido à pequena proporção de mulheres de cor preta e parda.

As pacientes deste estudo pertencem a faixas etárias avançadas devido às características de incidência do cancer de colo de útero e de endométrio nas mulheres.

O peso ideal, considerado para o cálculo da relação peso/peso ideal das falxas etárias mais avançadas do que as constantes na tabela do INSTITUTO DE RESSEGUROS DO BRASIL 4, foi a da maior faixa etária constante na tabela, pois, ao observarmos a tabela semelhante para homens, onde há faixas etárias mais avançadas, nota-se que há uma estabilização do peso ideal a partir da faixa etária de 55 a 59 anos.

$19,2 \%$ das pacientes foram classificadas no exame prévio ao tratamento no grupo com escaras, fato que não conseguimos explicação satisfatória, pois as pacientes eram mulheres e não prevíamos incidência de escaras no exame inicial ao plenajarmos este trabalho, deixando de incluir itens que pudessem explicar a origem dessas escaras. Esse grupo de pacientes não tinha características peculiares que as distinguissem das demais em relação à idade, relação peso/peso ideal e cor.

Ao observarmos a tabela 5 tivemos a impressão de que o número de horas do tratamento não influi no aparecimento de escaras ou na fase delas de modo apreciável. O número de casos desse trabalho não permitiu um estudo estatístico para comprovação dessa impressão.

Ao analisarmos a tabela 6, conseguimos distinguir dois grupos de pacientes quanto à incidência de escara. O primeiro grupo tem uma incidência de escara de $77,8 \%$, sendo constituído pelo grupo de mulheres com 60 anos ou mais, e um outro grupo com menor incidência de escara $(33,3 \%)$ constituído de mulheres com menos de 60 anos. Identificamos então a existência na população estudada de um grupo de maior risco para o desenvolvimento de escaras, aquele de mulheres com 60 anos ou mais. Dentre as que tiveram escara, as pacientes de faixa etária mais adiantada tiveram escara de fase mais avançada.

Análise semelhante feita na tabela 7 mostra também dois grupos de diferente incidência de desenvolvimento de escara. O grupo de maior incidência de escara $(66,7 \%)$ é o de mulheres com relação peso/peso ideal de $110 \%$ ou mais e o grupo de menor incidência de escara $(33,3 \%)$ é o constituído de mulheres com relação peso/peso ideal menor que $110 \%$. Constitui-se o grupo de mulheres com a relação peso/peso ideal de $110 \%$ ou mais no grupo de maior risco para o desenvolvimento de escara. Observou-se que, para pacientes com relação peso/peso ideal maior, foram encontradas escaras de fase mais avançada.

Combinando os dois fatores de risco de desenvolvimento de escara por nós sugeridos (relação peso/peso ideal dr $110 \%$ ou mais e faixa etária de 60 anos ou mais), constitui-se a tabela 8 . 
ANGARTEN, M.G. e CJlaboradora - Detecção de alterações em exame físico da pele da região de apoio de pacientes submetidos prolongadamente a um mesmo decúbito. Rev. Bras. Enf.; DF, 33 : 443-452, 1980.

\section{TABELA 8}

Distribuição das mulheres, segundo a presença ou ausêneia de escara, relação peso/peso ideal e idado

\begin{tabular}{|c|c|c|c|}
\hline \multirow{2}{*}{$\begin{array}{c}\text { peso/peso ideal } \\
(\%)\end{array}$} & \multirow[b]{2}{*}{ escara } & \multicolumn{2}{|c|}{ idade } \\
\hline & & $\begin{array}{l}\text { menor de } \\
60 \text { anos }\end{array}$ & $\begin{array}{l}\text { maior ou }=d e \\
60 \text { anos }\end{array}$ \\
\hline \multirow[t]{2}{*}{ menor de $110 \%$} & presença & 2 & 1 \\
\hline & ausëncia & 5 & 1 \\
\hline \multirow{2}{*}{$\begin{array}{l}\text { maior ou a } \\
110 \%\end{array}$} & presença & 2 & 6 \\
\hline & ausēncia & 3 & 1 \\
\hline
\end{tabular}

Da análise desta tabela resulta que, das 11 pacientes que desenvolveram escara, 9 delas pertencem ao grupo aqui identificado como o grupo de maior risco para o desenvolvimento de escara nesse estudo.

As outras 2 pacientes que desenvolveram escara tinham as seguintes características: a primeira tinha 49 anos e relação peso/peso ideal de $100 \%$ e desenvolveu escara de $1 .^{\text {a }}$ fase; a segunda tinha 45 anos e relação peso/peso ideal de $67 \%$ e desenvolveu escara de $3 .^{\text {a }}$ fase. A origem da escara dessa última paciente pode ser explicada pelo fato apontado por BERECEK ${ }^{1}$ de que as pessoas com baixo peso, quando comparadas com pessoas normais ou obesas, têm picos altos de pressão localizadamente quando deitadas. Uma relação peso/peso ideal baixa talvez se constitua em mais um fator de risco para o desenvolvi- mento de escara. No entanto, nossa casuística não permite tal afirmação.

\section{CONCLUSÃO}

Na população estudada foi encontrada uma incidência muito alta de escara de decúbito $(52,4 \%)$.

Houve alta incidência de pacientes admitidos com escara de decúbito $(19,2 \%)$.

As pacientes com 60 anos ou mais constituem um grupo de maior risco para desenvolvimento de escara $(77,8 \%$ de incidência de escara).

As pacientes com relação peso/peso ideal de $110 \%$ ou mais constituem um grupo de maior risco para desenvolvimento de escara $(66,7 \%$ de incidência de escara).

As escaras desenvolvidas durante o tratamento são mais avançadas quando consideradas pacientes mais idosas ou com relação peso/peso ideal maior. 
ANGARTEN, M.G. e Cslaboradora - Deteç̧ão de alterações em exame físico da pele da regiảo de apoio de pacientes submetidos prolongadamente a um mesmo decúbito. Rev. Bras. Enf.; DF, 33 : 443-452, 1980.

Dentre 11 pacientes que desenvolveram escara de decúbito, 9 pacientes $(81,8 \%)$ estavam no grupo de mulheres com 60 anos ou mais ou no grupo de relação peso/peso ideal de $110 \%$ ou mais.

\section{RECOMENDAÇÃO}

As autoras consideram importante a realização de estudos que apontem os métodos de prevenção de escaras mais adequados para pacientes submetidos a um mesmo decúbito prolongadamente.

\section{RESUMO}

As autoras estudaram 26 pacientes submetidas a radium moldagem. Encontraram incidência de escara de decúbito em $52,4 \%$ após esse tratamento. Identificaram como grupo de maior risco para o desenvolvimento de escara de decúbito as pacientes com 60 anos ou mais (incidência de $77,8 \%$ de escara de decúbito) e o grupo de pacientes com relação peso/peso ideal com $110 \%$ ou mais $(66,7 \%)$.

As autoras consideram importante a realização de estudos que apontem os métodos de prevenção de escaras mais adequados para pacientes submetidas a um mesmo decúbito prolongadamente.

\section{BIBLIOGRAFIA}

1. BERECEK, K. H. - Etiology of decubitus ulcers. Nurs. Clin. N. Amer., 10 (1) : 157-79, 1975.

2. COMARÚ, M. N. et CAMARGO, C. A. Um problema de enfermagem - as escaras de decúbito. Rev. Bras. Enf., 24 (6) : 96-106, 1971.

3. GERSON, L. W. - The incidence of pressure sores in active treatment hospitals. Int. J. Nurs. Stud., 12: 201-4, 1975.

4 INSTITUTO DE RESSEGUROS DO BRASIL, Rio de Janeiro - Julga- mento médico em seguro-vida. $2 .^{\mathrm{a}}$ ed. Rio de Janeiro, Consultoria do Instituto de Resseguros do Brasil, 1959.

5. LOWTHIAN, P. - Pressure sore prevalence - a survey of sores in orthopaedic patients. Nurs. Times, 75 (11) 358-60, 1979.

6. RUBIN, C. F., DIETZ, R. R. EABRUZZESE, R.S. - Auditing the decubitus ulcer problem. Am. J. Nurs., 74 (10) : 1820-1, 1974.

7. WILLIAMS, A. - A study of factors contributing to skin break down. Nurs. Res., 21 (3) :238-43, 1972. 